\title{
QQ VUL REVISITED
}

\author{
M. S. CATALÁN ${ }^{1,2}$, S. D. DAVEY ${ }^{2}$, R. C. SMITH ${ }^{1}$, \\ D. H. P. JONES ${ }^{3}$ \\ 1. Dept. of Physics and Astronomy, University of St Andrews, \\ Fife KY16 9SS, UK \\ 2. Astronomy Centre, Division of Physics and Astronomy, \\ University of Sussex, Brighton, BN1 9QH, UK \\ 3. RGO, Madingley Rd., Cambridge, CB3 OEZ, UK
}

\begin{abstract}
With simultaneous blue $(\lambda \lambda 4200-5000 \AA)$ and far-red $(\lambda \lambda 7950-$ $8380 \AA$ ) phase-resolved, high resolution spectrophotometry, we re-examine the magnetic CV QQ Vul. A thorough radial velocity and flux analysis of all the observable features is carried out. We derive a complete set of system parameters and classify the donor star to be a spectral type M4.5 V. In addition to the Balmer, He I and He II emission lines in the blue range, arising from the accretion regions, we detect various emission features which solely follow the movement of the secondary star. The lines, which we interpret as reprocessed light from the illuminated hemisphere of the donor star, are Mg II at $\lambda 4481 \AA$, He II at $\lambda 8236 \AA$ and $\mathrm{C}_{\text {I }}$ at $\lambda 8335 \AA$. Intensity maps based on the line flux and radial velocity variations of the $\mathrm{Na} I$ absorption near $\lambda 8190 \AA$ and $\mathrm{Mg}$ II emission feature were also made. These show that the line distribution on the surface of the donor is consistent with being symmetric. As a result of our analysis the position of the real zero phase was found to be at $\phi=0.42$ of the linear polarisation ephemeris.
\end{abstract}

\section{Introduction}

QQ Vul is a long-period (222.5 m) AM Her type CV first detected with the HEAO-1 low-energy detectors (Nugent et al. 1983). Since its discovery, quite a number of studies have been published on this object in the optical and X-ray wavelengths (e.g. Nousek et al. 1984; Mukai \& Charles 1987; Osborne et al. 1987). However, to date, its system parameters are still poorly known. We report recent spectroscopic observations of QQ Vul, paying particular attention to features that originate from the secondary, and derive its system parameters.

A. Evans and J. H. Wood (eds.), Cataclysmic Variables and Related Objects, 227-228.

O 1996 Kluwer Academic Publishers. Printed in the Netherlands. 

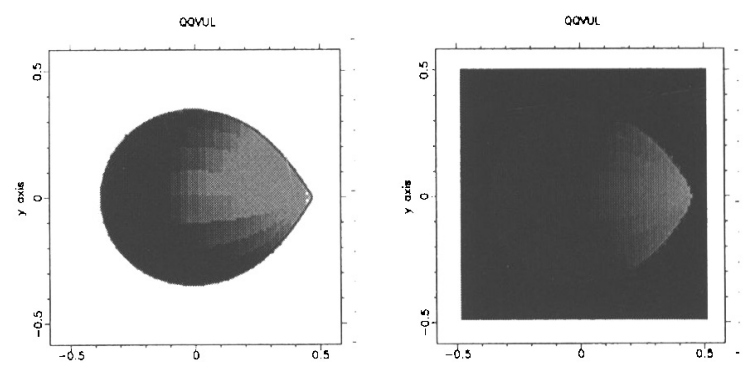

Figure 1. Intensity distribution of the $\mathrm{Na}$ I (left) and $\mathrm{Mg}$ II (right) on the secondary star.

\section{Intensity maps and the spectral type of the secondary}

Our spectral analysis has revealed that the emission spectrum seen is heavily peppered with lines that arise from the heated face of the donor star. In particular we found that the $\mathrm{Mg}$ II doublet at $\lambda 4481 \AA$, He II at $\lambda 8236 \AA$ and $\mathrm{C}_{\mathrm{I}}$ at $\lambda 8335 \AA$ have radial velocities and line flux variations consistent with an irradiation scenario. By adopting the same technique as in Davey \& Smith (1992), we have obtained $\mathrm{NaI}$ and Mg II intensity maps of the donor star. Those derived from the line flux variations are shown in Fig. 1. Using the $\mathrm{TiO}$ band ratios method (Wade \& Horne 1988), we have found the spectral type of the donor star in QQ Vul to be an M4.5 V.

\section{Dynamical determination of the system parameters}

The system parameters were found using the $v \sin (i)$ technique (see e.g. Martin et al. 1989). A preliminary estimate of the secondary star's true $K$-velocity was obtained from the average of the velocity semi-amplitudes derived from the $\mathrm{NaI}$ absorption feature, formed on the unirradiated regions of the star, and the $\mathrm{Mg}$ II emission originating from its illuminated side. We found $K_{2}=174 \pm 12 \mathrm{~km} \mathrm{~s}^{-1}$. Combined with our measurement of the rotational velocity $v_{\text {abs }} \sin (i)=112 \pm 10 \mathrm{~km} \mathrm{~s}^{-1}$ from the Na I lines, the mass ratio was found to be $q=0.79 \pm 0.17$. This, in turn, yields a primary mass of $M_{1}=0.42 \pm 0.18 \mathrm{M}_{\odot}$ and a secondary mass $M_{2}=0.33 \pm 0.18 \mathrm{M}_{\odot}$.

\section{References}

Davey, S.C., Smith , R.S., 1992, MNRAS, 257, 476

Martin J.S., Friend, M.T., Smith, R.C., et al.,1989, MNRAS, 240, 519

Mukai, K., Charles, P.A., 1987, MNRAS, 226, 209

Nousek, J.A., Takalo, L.O., Schmidt, G.D., et al., 1984, Ap. J., 277, 682

Nugent, J.J., Jensen, K.A., Nousek, J.A., et al., 1983, Ap. J. Supp., 51, 1

Osborne, J.P., Beuermann, K., Charles, P., et al., 1987, Ap. J., 315, L123

Wade, R.A., Horne, K., 1988, Ap. J., 324, 411 